\title{
Efficacy and tolerability of a fixed-dose moxifloxacin - dexamethasone formulation for topical prophylaxis in LASIK: a comparative, double-masked clinical trial
}

\author{
Mauro Campos \\ Mariana Ávila \\ Anelise Wallau \\ Cristina Muccioli \\ Ana Luisa Höfling-Lima \\ Rubens Belfort Jr \\ Department of Ophthalmology, Escola \\ Paulista de Medicina, Universidade \\ Federal de São Paulo, São Paulo, Brazil
}

Purpose: To compare the efficacy and tolerability of a fixed-dose combination of $0.5 \%$ moxifloxacin and $0.1 \%$ dexamethasone formulation (MFLX/DEX) vs conventional dosing with both agents dosed separately for prophylaxis after laser-assisted in situ keratomileusis (LASIK).

Methods: A prospective, randomized, double-masked, parallel-group study of 64 patients undergoing bilateral LASIK. Patients received either combined MFLX/DEX and placebo or moxifloxacin and dexamethasone dosed separately in both eyes. Baseline and postoperative assessments were made on surgery days $-2,1,3,8$, and 15 and consisted of uncorrected visual acuity (UCVA), intraocular pressure (IOP), severity of inflammation, endothelial cell loss, ocular pain, burning, and itching sensation. The posterior segment was evaluated at the screening and exit visits.

Results: Of the 64 patients treated, 7 eyes did not meet the inclusion criteria and were excluded from the analysis. No ocular infection or persistent inflammation developed. Postoperatively there were no statistical differences between treatments for most parameters measured. More eyes in the combined MFLX/DEX group reported pruritus and burning post operatively; however, differences were also observed at baseline.

Conclusion: Topical prophylaxis with MFLX/DEX eye drops was well tolerated and is therapeutically equivalent to conventional dosing with moxifloxacin and dexamethasone from individual bottles.

Keywords: moxifloxacin, dexamethasone, LASIK, prophylaxis

\section{Introduction}

Laser-assisted in situ keratomileusis (LASIK) is the most popular operation for refractive correction in developed countries and one of the most common elective surgical procedures worldwide (Kalorama Information 2007). The surgical risks are low, there is little eye pain or redness postoperatively, and the visual rehabilitation is very fast. LASIK patients are usually prescribed antibiotic and steroid eye drops for the first 5-14 days after surgery to prevent infection and minimize inflammation, along with ad lib use of preservative-free moisturizing eye drops to combat dry eye. Compliance with prophylactic medications is critical to reduce the risk of serious complications, such as infectious keratitis.

Alcon Laboratories, Inc. has developed a new antibiotic-steroid formulation consisting of $0.5 \%$ moxifloxacin and $0.1 \%$ dexamethasone (MFLX/DEX) (Vigadexa $^{\circledR}$, Alcon Laboratories, Inc., Fort Worth, TX, USA). We tested this combination therapy in a typical LASIK population and compared its efficacy 
and tolerability vs conventional administration of $0.5 \%$ moxifloxacin (Vigamox ${ }^{\circledR}$, Alcon Laboratories, Inc.) and $0.1 \%$ dexamethasone (Maxidex ${ }^{\circledR}$, Alcon Laboratories, Inc.) from individual bottles.

\section{Patients and methods}

A total of 64 consecutive patients who presented for LASIK correction were randomly assigned to receive either the fixed-dose combination of MFLX/DEX formulation and a dextran-methylcellulose placebo (Lacrima Plus ${ }^{\circledR}$, Alcon Laboratories, Inc.) or conventional dosing with $0.5 \%$ moxifloxacin and $0.1 \%$ dexamethasone solutions in individual bottles. The combination MFLX/DEX formulation contained moxifloxacin hydrochloride, dexamethasone sodium phosphate, edetate disodium (chelating agent), sodium chloride, boric acid, sorbitol, and tyloxapol (as surfactant).

Group assignment was masked from the patients as well as the researchers and clinic staff. Sixty-one patients (121 eyes) met the protocol inclusion/exclusion criteria, including 60 eyes randomized to the combination agent and 61 eyes randomized to separate dosing of both drugs. The study protocol was approved by an independent institutional review board and informed consent was obtained from each patient prior to participation in the study.

The protocol inclusion criteria consisted of men and women of any race who were 18-50 years old, had either myopia of up to $-8.0 \mathrm{D}$ or hyperopia of up to $+4.0 \mathrm{D}$, with or without astigmatism of up to $3.0 \mathrm{D}$, and whose dilated funduscopy at the time of enrollment was within normal limits. Patients were excluded from the study if they had (a) used topical or systemic steroids or nonsteroidal anti-inflammatory drugs (NSAID) in the 14 days before enrollment (except aspirin, which was allowed up to $100 \mathrm{mg}$ daily); (b) used ocular antimicrobial drugs within 30 days of enrollment; (c) active inflammation on slit-lamp biomicroscopy or history of chronic or recurrent ocular inflammatory disease; (d) significant ocular pain; (e) uncontrolled glaucoma or intraocular hypertension; (f) uncontrolled diabetes mellitus, proliferative diabetic retinopathy, or moderately severe nonproliferative diabetic retinopathy; $(\mathrm{g})$ known or suspected allergy to any of the drugs or preservatives to be used in the study; (h) active epithelial herpes simplex, vaccinia, chicken pox, corneal or conjunctival disease, ocular tuberculosis or fungal disease; (i) intraocular pressure measurements greater than $20 \mathrm{mmHg}$; (j) participated in a clinical trial within 12 months of enrollment; (k) any severe systemic disease or condition that made self-treatment with eye drops difficult; (1) sight in a single eye; $(\mathrm{m})$ or best-corrected visual acuity (BCVA) worse than 1.0 on the $\log$ MAR scale. Pregnant or lactating women or women of childbearing age not using reliable birth control methods were also excluded. Patients who were receiving a topical prostaglandin for IOP control were instructed to discontinue its use at least 4 days prior to surgery and until the study was completed. All surgical candidates were told to stop wearing contact lenses for at least 2 weeks before the LASIK procedure.

Bilateral, simultaneous, wavefront-guided LASIK was performed using the LADARVision system (Alcon Laboratories, Inc.). Patients in the combined MFLX/DEX treatment group received two opaque bottles, one containing the moxifloxacin-dexamethasone solution and the other placebo. Patients in the control group also received two opaque bottles, one bottle containing moxifloxacin and the other dexamethasone. All patients were instructed to apply 1 drop from each bottle in both eyes 4 times a day, waiting 5 minutes between drops, beginning 15 minutes after completion of the procedure and continuing until the 15 th day.

Two days before surgery (Day -2 ), the baseline values were recorded for visual acuity (VA), IOP, number of cells in the anterior chamber, clinical impression of inflammation of eyelids and conjunctiva, ocular pain, burning or itching sensation, and fundus status. These assessments were repeated on the first day after surgery (Day 1) and thereafter on days 3, 8, and 15, except for dilated funduscopy, which was performed only at the screening and exit visits.

The visual acuity was determined with an ETDRS or modified ETDRS chart and expressed in $\log$ MAR values. Preoperatively, all patients had a BCVA of $0.0 \log$ MAR, while postoperatively the vision was measured uncorrected. The IOP readings were taken with the Goldmann Applanation Tonometer. Slit-lamp biomicroscopy was used to count the number of cells in the anterior chamber; a score of $0 \leq 5$ cells; $1=5-10$ cells; $2=11-20$ cells; $3=21-50$ cells; and $4 \geq 50$ cells. Any visible sign of active ocular inflammationredness, swelling, tearing, or discharge - was documented. The patients were asked to subjectively rank their eye pain on a 5 -point scale, from $0=$ none to $5=$ severe. Sensations of ocular burning and pruritus were recorded as yes or no. The fundus was visualized through a dilated pupil on Day -2 and Day 15. All evaluations were made binocularly.

Seven eyes were excluded from the per-protocol analysis: 6 eyes of 3 patients older than 50 years and 1 eye of 1 patient whose astigmatism was higher than 3 diopters. Statistical analysis was performed on the remaining 121 eyes by means of Mann-Whitney and chi-square tests, non-paired t tests, and 
variance analysis (ANOVA) with two-tailed testing at a 5\% confidence level $(\mathrm{p}=0.05)$.

\section{Results}

At randomization the treatment groups were similar for age, sex, and race. Seven eyes of 4 patients were excluded from the per protocol analysis. Demographics for the remaining 61 patients (121 eyes) are presented in Table 1. Both groups were matched for gender and race. The median age of patients in the combined MFLX/DEX group was slightly lower than in the separate moxifloxacin and dexamethasone group. There were more women than men in our study population. Two patients ( 3 eyes) in the moxifloxacin and dexamethasone separate group experienced surgical problems. One patient had minor bleeding in both eyes during the LASIK procedure and 1 patient had a thin flap in the left eye. All eyes in the combined treatment group were myopic and had astigmatism. Among those receiving separate antibiotic and anti-inflammatory eye drops, 29 eyes were myopic, 3 were hyperopic, and 27 had astigmatism. The maximum refractive error allowed in the study was $-8.0 \mathrm{D}$ to +4.0 D with up to $3.0 \mathrm{D}$ of cylinder. All evaluable patients (121 eyes) remained in the study through the Day 15 exit visit.

Both groups had similar postoperative improvement in visual acuity. The median UCVA at each visit was no different between treatments. A median UCVA of -0.10 and -0.06 on the $\log$ MAR scale was reported for the MFLX/DEX combined and the moxifloxacin and dexamethasone separate groups, respectively, by Day 15 .

Table I Patient demographics

\begin{tabular}{|c|c|c|c|}
\hline & $\begin{array}{l}\text { MFLX/DEX } \\
\text { combined } \\
\mathbf{N}=30\end{array}$ & $\begin{array}{l}\text { MFLX and DEX } \\
\text { separate } \\
\mathbf{N}=31\end{array}$ & \\
\hline \multicolumn{4}{|l|}{ Age (years) } \\
\hline Mean & 29.1 & 32.9 & \\
\hline Median & 28.5 & 31.0 & $P=0.033$ \\
\hline \multicolumn{4}{|l|}{ Gender } \\
\hline Female $^{\mathrm{a}}$ & 22 & 22 & \\
\hline Male & 8 & 9 & $P=0.8368$ \\
\hline \multicolumn{4}{|l|}{ Race } \\
\hline Caucasian ${ }^{\mathrm{b}}$ & 25 & 25 & \\
\hline African & 1 & 0 & \\
\hline Asian & 2 & 2 & \\
\hline Mulatto & 2 & 4 & $P=0.7848$ \\
\hline \multicolumn{4}{|l|}{$\begin{array}{l}\text { Surgical } \\
\text { problems }\end{array}$} \\
\hline No & 30 & 29 & \\
\hline Yes & 0 & $2^{c}$ & \\
\hline
\end{tabular}

${ }^{\mathrm{a} F e m a l e ~ v s ~ m a l e, ~} \chi^{2}=0.04$

${ }^{\mathrm{b}}$ Caucasian vs others, $\chi^{2}=0.07$.

'One patient had minor bleeding in both eyes and one patient had a thin flap in the left eye.
The intraocular pressure fluctuated curiously throughout the study, sometimes within the same eyes in a 48-hour period. When the median intraocular pressure was calculated, however, there was no statistical difference between the treatment groups. For the baseline visit and first week after surgery (up to Day 8), the IOP ranged from a minimum of $7 \mathrm{mmHg}$ to a maximum of $18 \mathrm{mmHg}$. On Day 15, an IOP spike to $28 \mathrm{mmHg}$ was recorded in both eyes of 1 patient in the combined MFLX/DEX treatment group. The patient was started on timolol every 12 hours and rechecked 6 days later, at which time the IOP was $10 \mathrm{mmHg}$ and the timolol was stopped. Off medication, her intraocular pressure remained within normal limits on subsequent visits.

As expected, there was no endothelial cell loss secondary to the keratomileusis. Fewer than 5 cells were recorded in the anterior chamber at any time regardless of treatment. Clinical examination was also negative for any injection or evidence of inflammation in the eyelids, conjunctiva, and sclera. On Day 1, 3 eyes in the combined MFLX/DEX group and 5 eyes in the moxifloxacin and dexamethasone separate group showed minor structural changes, infiltrate, or edema on biomicroscopy. These findings persisted in 1 eye for the first week, but by Day 15 all corneas were clear.

With regard to eye pain, no statistically significant differences were observed between the MFLX/DEX combined and the moxifloxacin and dexamethasone separate groups. Ocular pain was rated as "none", "minimal", or "mild", except for a single eye in the combined MFLX/DEX group that was reported as "moderately severe" on Day 8 (Figure 1). Careful examination revealed no obvious cause for the pain, which was relieved without analgesics within a few hours.

A burning sensation was reported in more study eyes in the MFLX/DEX combined group compared with the moxifloxacin and dexamethasone separate group at Day 1 (12 eyes vs 1 eye, respectively), but at baseline more eyes had ocular burning in the combined MFLX/DEX group. At Day 15, both groups had equal numbers (8) of eyes with burning (Figure 2). More patients who received combination MFLX/DEX therapy complained of itching eyes prior to LASIK than in the moxifloxacin and dexamethasone separate group (16 eyes vs 0 eyes, $\mathrm{p}<0.001$, respectively). This difference between treatment groups persisted following surgery and was statistically significant on Day 1, and Day 15 postoperative (15 eyes vs 3 eyes at Day 1, $\mathrm{p}<0.0019$, and 15 eyes vs 4 eyes at Day 15, p $<0.0053$, for the combined MFLX/DEX group and moxifloxacin and dexamethasone separate group, respectively) (Figure 3). When the data were analyzed excluding patients with pruritus at baseline, there were no differences between treatment groups in postoperative ocular pruritus (Day 1, 7 eyes vs 3 eyes; Day 3, 10 eyes 
- Group $1^{*}(\mathrm{~N}=60) \square \operatorname{Group} 2^{* *}(\mathrm{~N}=61)$

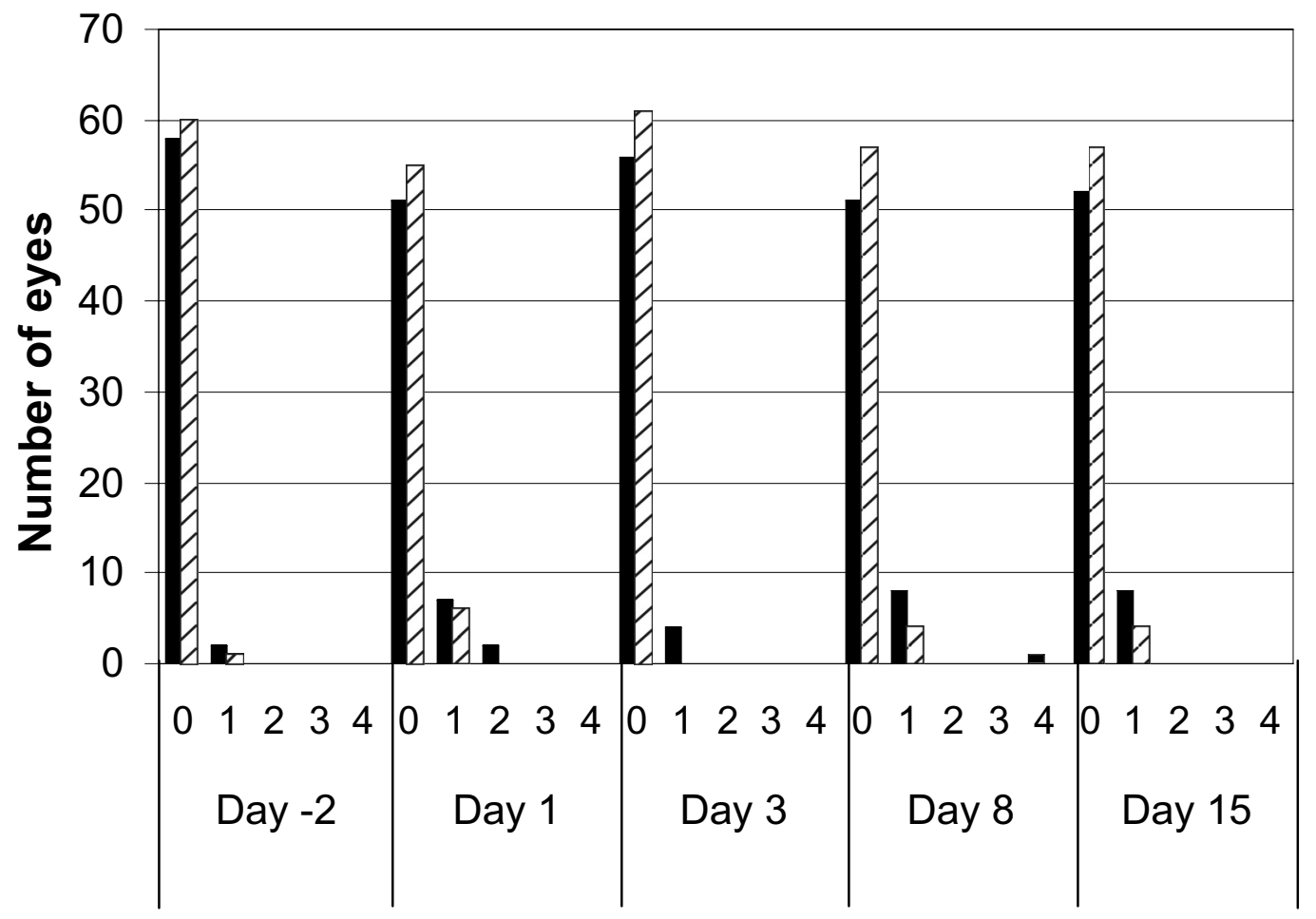

\section{Day in relation to surgery}

Pain Scale:

$0=$ none

$1=$ minimal sensation of discomfort

2 = mild, tolerable eye pain

$3=$ moderate, requiring the use of over-the-counter analgesics

$4=$ moderately severe, more lasting pain requiring use of another analgesic agent

5 = severe, intense periocular or irradiating ocular pain

*Group 1 = combined moxifloxacin-dexamethasone eye drops

${ }^{* *}$ Group 2 = separate moxifloxacin and dexamethasone solutions

Figure I Ocular pain by treatment group. Comparison of ocular pain scores at baseline (Day -2$)$ and each postoperative visit for MFLX/DEX combined and MFLX and DEX dosed separately.

vs 12 eyes; Day 8, 2 eyes vs 6 eyes; and Day 15, 11 eyes vs 4 eyes; for the combined MFLX/DEX and moxifloxacin and dexamethasone separate groups, respectively) ( $p>0.05)$.

Dilated funduscopy showed normal vitreous, retina, macula, and choroid of all eyes at all times. There were no adverse events reported related to study medication.

\section{Discussion}

Ocular infection is a dreaded complication of any ophthalmic procedure, particularly after an elective surgery such as LASIK. Although endophthalmitis is fortunately rare in LASIK and infectious keratitis has a low incidence (up to 1.5\%) (Chang et al 2004; Solomon et al 2003), infection of any kind can have devastating consequences to visual function and every step should be taken to prevent it (Solomon and Donnenfeld 2005). The majority of keratitis cases are noninfectious and are diagnosed as diffuse lamellar keratitis (DLK) (Moshirfar et al 2007). DLK is characterized by an ill-defined, widespread inflammatory reaction at the flap interface (Alio et al 2000) and diffuse, multifocal, sterile 


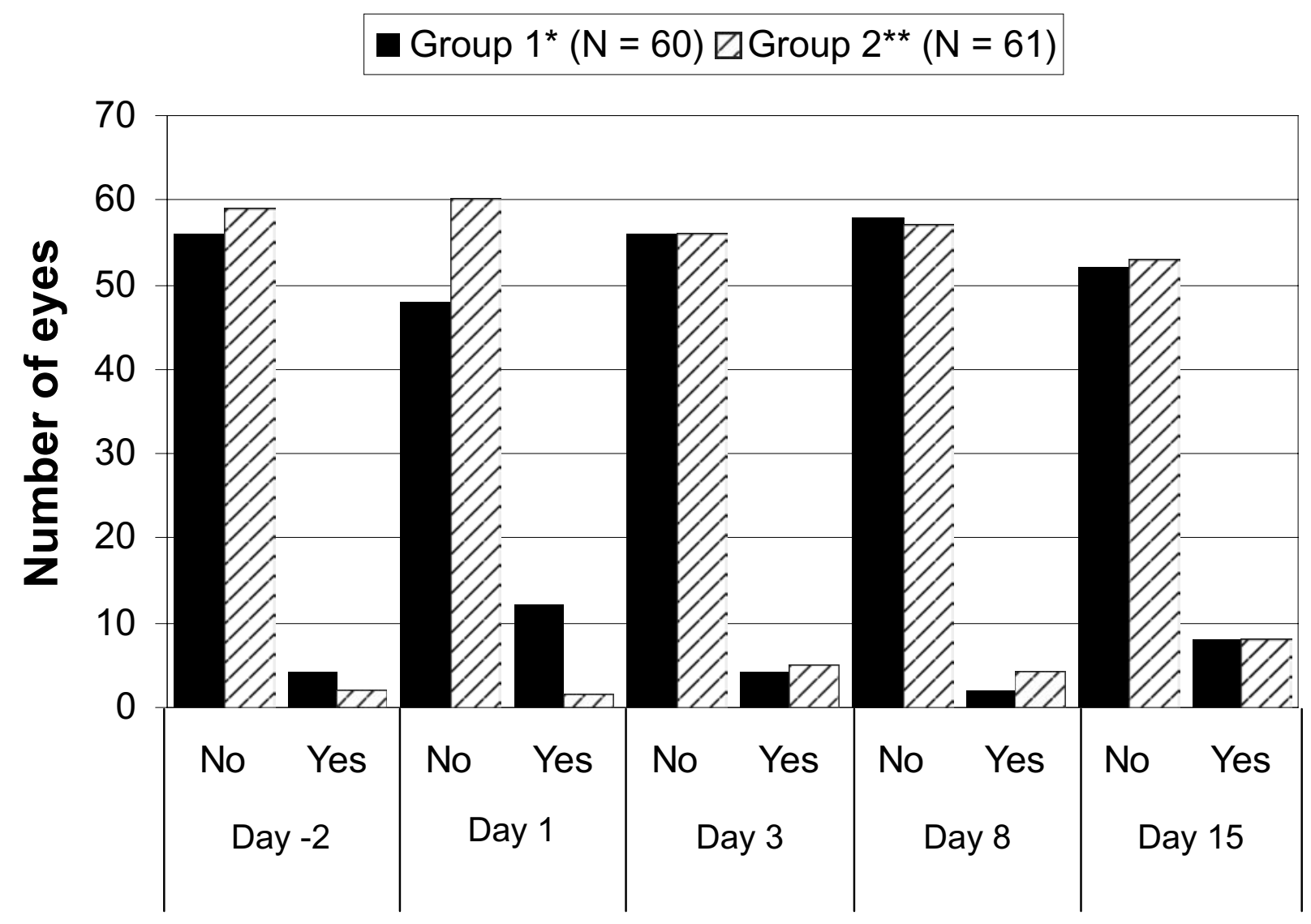

\section{Day in relation to surgery}

*Group 1 = combined moxifloxacin-dexamethasone eye drops

${ }^{*}$ Group 2 = separate moxifloxacin and dexamethasone solutions

Figure 2 Burning/stinging sensation in eye by treatment group. Comparison of burning/stinging scores at baseline (Day -2$)$ and each postoperative visit for MFLX/DEX combined and MFLX and DEX dosed separately. Burning and stinging scores in each eye were rated as either "yes" or "no".

corneal infiltrates (Smith and Maloney 1998; Lifshitz et al 2005). DLK shows a strong association with epithelial defects and anterior chamber reactions developing after LASIK (Shah et al 2000; Johnson et al 2001; Chang et al 2004; Moilanen et al 2004).

Many practitioners opt for peri-LASIK prophylaxis with an antibiotic solution - usually a fourth-generation fluoroquinolone (Solomon and Donnenfeld 2005) - and a topical steroid, such as prednisolone or dexamethasone, to control the initial corneal inflammation that sometimes leads to more serious keratitis. Patients are typically instructed to apply the eye drops at 5-minute intervals 4 times a day for at least 5 days after LASIK.

Several studies have established the safety and effectiveness of moxifloxacin in the prevention and treatment of infectious bacterial keratitis, including that of mycobacterial origin (Kowalski et al 2003; Abshire et al 2004; Lee et al 2005;
Durrie and Trattler 2005; Yee et al 2006; Solomon et al 2007). Likewise, dexamethasone has a long and reliable history as a useful anti-inflammatory adjuvant in cataract surgery (van Endt et al 1997; Notivol et al 2004; Russo et al 2007) with no adverse effect on the corneal epithelium (Gris et al 2004; Yulek et al 2006). The present study evaluated a fixed-dose combination product of moxifloxacin and dexamethasone in patients undergoing LASIK.

We monitored patients who underwent bilateral LASIK to check for signs of infection and inflammation under two drug regimens; half of the patients were given eye drops containing a fixed-dose combination of $0.5 \%$ moxifloxacin with $0.1 \%$ dexamethasone and a placebo and half received same-strength moxifloxacin and dexamethasone in the conventional manner from individual bottles. No infection developed and there was no corneal inflammation beyond the initial postoperative period. Results for UCVA, IOP, 


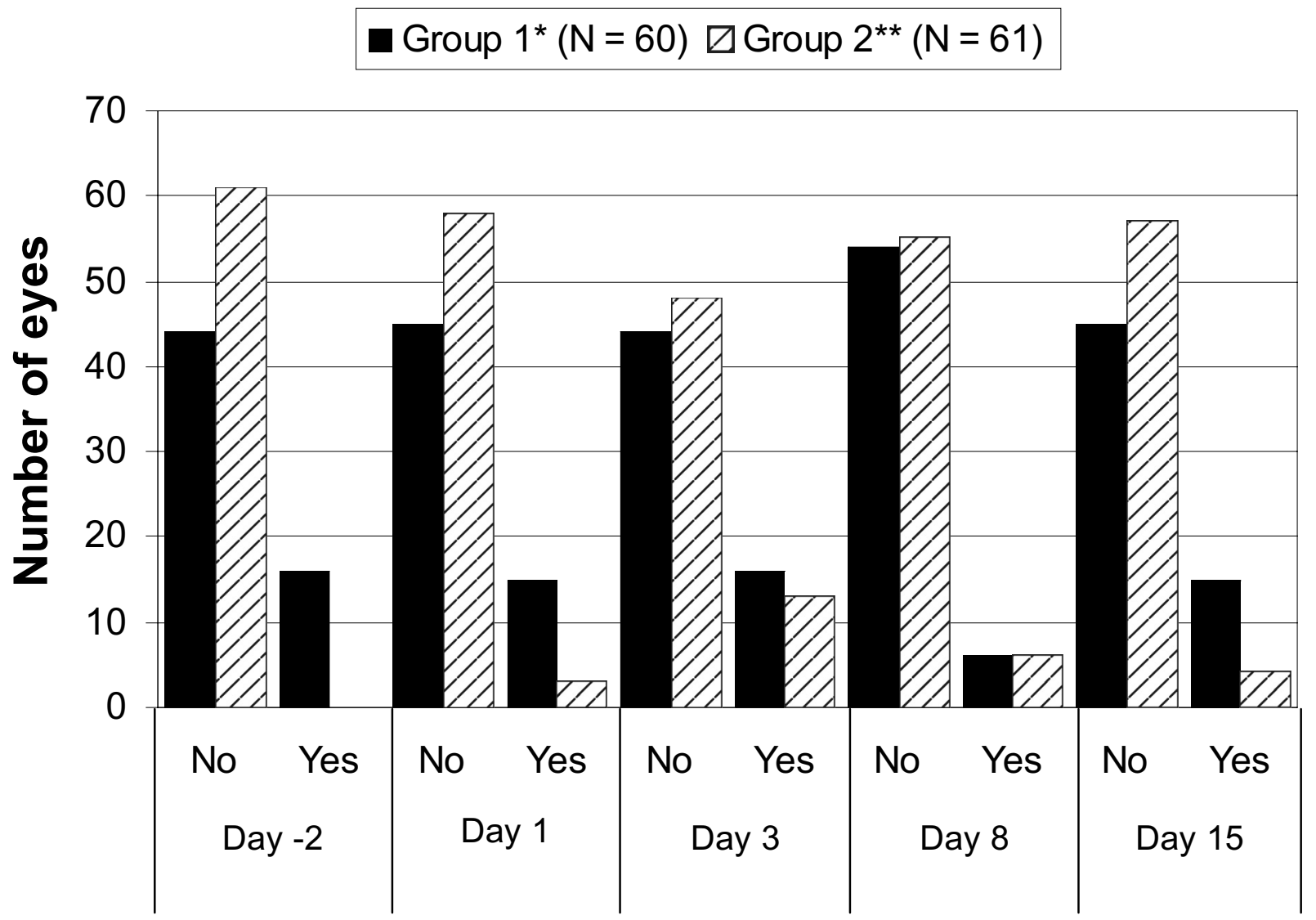

\section{Day in relation to surgery}

\section{*Group 1 = combined moxifloxacin-dexamethasone eye drops \\ **Group 2 = separate moxifloxacin and dexamethasone solutions}

Figure 3 Ocular itching by treatment group. Comparison of itching scores at baseline (Day -2$)$ and each postoperative visit for MFLX/DEX combined and MFLX and DEX dosed separately. Ocular itching scores in each eye were rated as either "yes" or "no".

endothelial cell count, slit lamp, and fundus evaluation were similar for patients treated with the combination eye drops or the separate medications. One patient had an isolated rise in intraocular pressure bilaterally that was unexplained. After a short course of timolol, her IOP returned to normal levels and has remained in the $10-12 \mathrm{mmHg}$ range over the ensuing months.

The combination of moxifloxacin and dexamethasone in a single vehicle simplifies eye-drop application and is expected to improve patient compliance with treatment (Ermis et al 2004). From an economical standpoint, the combination product reduces medication cost since patients only need to purchase a single bottle of medication instead of two separate bottles.

Decreasing the complexity of dosing and the number of separately administered ophthalmic medications is likely to decrease dosing errors and noncompliance following LASIK.
Compliance with topical ocular glaucoma medications has been shown to decrease when patients were changed from a single medication regimen to a regimen of two medications (Patel and Spaeth 1995). Similarly, the addition of a second non-once-daily medication was also associated with an increase in percentage of dosage errors (37\%) in a short-term (60 days) adherence study in glaucoma patients (Robin et al 2007). Although one may argue that patient compliance following LASIK surgery differs because the treatment course is shorter and there is greater patient motivation for this elective procedure, the prophylactic drug regimens following LASIK surgery are more complex than those for glaucoma treatment, increasing the likelihood of inadvertent dosing errors.

The use of a combination product has also been shown to positively impact clinical outcomes. For example, greater IOP lowering was demonstrated with fixed-dose combined dorzolamide-timolol drops compared with both agents 
dosed separately. Greater efficacy was attributed to better patient compliance and/or to decreased medication washout (Choudhri et al 2000).

Medication washout occurs when more than one topical ocular medication is dosed at the same time of day. Administering a second topical ocular product too close in time to the first can reduce the amount of the first drug delivered to the eye, such that if a patient waits less than 5 minutes between medications, a significant proportion is washed out by the second (Chrai et al 1974). Given that LASIK patients must administer two or three different prophylactic ocular medications at similar dosing intervals, the time to administer these agents (including waiting time) can be as long as 15 minutes.

Certain findings from the present study remain unexplained. A single patient in the combined MFLX/DEX treatment group reported moderately severe pain in 1 eye on Day 8. We could find no physical cause for the pain and it resolved within 24 hours without specific treatment. Two weeks after surgery, more patients in both treatment groups complained of ocular discomfort than before the procedure, though the sensation was ill-defined and only minimally uncomfortable. LASIK, like all eye surgeries, involves controlled trauma to the ocular tissues followed by a period of recovery and healing. In this study, regardless of treatment group, only a small percentage of patients reported mild discomfort

More patients taking the combination MFLX/DEX drops complained of itching after LASIK. Upon further inspection of the data it was determined that these were the same patients who complained of ocular pruritus before the LASIK procedure, and we believe this finding reflects the cohort's idiosyncrasies, independent of the medication received. There were no adverse reactions related to either drug regimen and all patients appeared to tolerate the eye drops very well.

\section{Conclusion}

A fixed-dose formulation of moxifloxacin and dexamethasone was found to be safe and effective in preventing infection and controlling inflammation post-LASIK. The combined antibiotic-anti-inflammatory eye drop solution was therapeutically equivalent to conventional therapy with individual bottles of moxifloxacin and dexamethasone in this population.

\section{Acknowledgments}

The authors would like to acknowledge Grace Darling for medical writing contributions.

\section{Disclosures}

The authors have no financial interest in any of the products in this study, which was supported in part by Alcon Laboratories, Inc., Fort Worth, Texas, USA.

Trademarks are property of their respective owners.

\section{References}

Abshire R, Cockrum P, Crider J, et al. 2004. Topical antibacterial therapy for mycobacterial keratitis: potential for surgical prophylaxis and treatment. Clin Ther, 26:191-6.

Alio JL, Perez-Santonja JJ, Tervo T, et al. 2000. Postoperative inflammation, microbial complications, and wound healing following laser in situ keratomileusis. J Refract Surg, 16:523-38.

Chang MA, Jain S, Azar DT. 2004. Infections following laser in situ keratomileusis: an integration of the published literature. Surv Ophthalmol, 49:269-80.

Choudhri S, Wand M, Shield BM. 2000. A comparison of dorzolamidetimolol combination versus the concomitant drugs. Am J Ophthalmol, 130:832-33.

Chrai SS, Makoid MC, Eriksen SP, et al. 1974. Drop size and initial dosing frequency problems of topically applied ophthalmic drugs. J Pharm Sci, 63:333-8.

Durrie D, Trattler W. 2005. A comparison of therapeutic regimens containing moxifloxacin $0.5 \%$ ophthalmic solution and gatifloxacin $0.3 \%$ ophthalmic solution for surgical prophylaxis in patients undergoing LASIK or LASEK. J Ocul Pharmacol Ther, 21:236-41.

Ermis SS, Aktepe OC, Inan UU, et al. 2004. Effect of topical dexamethasone and ciprofloxacin on bacterial flora of healthy conjunctiva. Eye, 18:249-52.

Gris O, Guell JL, Wolley-Dod C, et al. 2004. Diffuse lamellar keratitis and corneal edema associated with viral keratoconjunctivitis 2 years after laser in situ keratomileusis. J Cataract Refract Surg, 30:1366-70.

Johnson JD, Harissi-Dagher M, Pineda R, et al. 2001. Diffuse lamellar keratitis: incidence, associations, outcomes, and a new classification system. J Cataract Refract Surg, 27:1560-6.

Kalorama Information.com. Refractive vision correction: a market analysis [online]. E-pub Mar 1, 2007:41 pages. URL: http://www. kaloramainformation.com/Refractive-Vision-Correction-1445485/.

Kowalski RP, Dhaliwal DK, Karenchak LM, et al. 2003. Gatifloxacin and moxifloxacin: an in vitro susceptibility comparison to levofloxacin, ciprofloxacin, and ofloxacin using bacterial keratitis isolates. $\mathrm{Am} \mathrm{J}$ Ophthalmol, 136:500-5.

Lee SB, Oliver KM, Mohan SK, et al. 2005. Fourth-generation fluoroquinolones in the treatment of mycobacterial infectious keratitis after laserassisted in situ keratomileusis surgery. Can J Ophthalmol, 40:750-3.

Lifshitz T, Levy J, Mahler O, Levinger S. 2005. Peripheral sterile corneal infiltrates after refractive surgery. $J$ Cataract Refract Surg, $31: 1392-5$.

Moilanen JA, Holopainen JM, Helinto M, et al. 2004. Keratocyte activation and inflammation in diffuse lamellar keratitis after formation of an epithelial defect. J Cataract Refract Surg, 30:341-9.

Moshirfar M, Welling JD, Feiz V, et al. 2007. Infectious and noninfectious keratitis after laser in situ keratomileusis. Occurrence, management, and visual outcome. J Cataract Refract Surg, 33:474-3.

Notivol R, Bertin D, Amin D, et al. 2004. Comparison of topical tobramycin-dexamethasone with dexamethasone-neomycin-polymyxin and neomycin-polymyxin-gramicidin for control of inflammation after cataract surgery: results of a multicenter, prospective, three-arm, randomized, double-masked, controlled, parallel-group study. Clin Ther, 26:1274-85.

Patel SC, Spaeth GL. 1995. Compliance in patients prescribed eye drops for glaucoma. Ophthalmic Surg, 26:233-6.

Robin AL, Novack GD, Covert DW, et al. 2007. Adherence in glaucoma: objective measurements of once-daily and adjunctive medication use. Am J Ophthalmol, 144:533-40. 
Russo S, Papa V, Di Bella A, et al. 2007. Dexamethasone-netilmicin: a new ophthalmic steroid-antibiotic combination. Efficacy and safety after cataract surgery. Eye, 21:58-64.

Shah MN, Misra M, Wilhelmus KR, et al. 2000. Diffuse lamellar keratitis associated with epithelial defects after laser in situ keratomileusis. J Cataract Refract Surg, 26:1312-18.

Smith RJ, Maloney RK. 1998. Diffuse lamellar keratitis: A new syndrome in lamellar refractive surgery. Ophthalmology, 105:1721-6.

Solomon R, Donnenfeld ED. 2005. Changing patterns of infection after LASIK. Ther Updates Ophthalmol, 6:3, 8.

Solomon R, Donnenfeld ED, Azar DT, et al. 2003. Infectious keratitis after laser in situ keratomileusis: Results of an ASCRS survey. J Cataract Refract Surg, 29:2001-6.
Solomon R, Donnenfeld ED, Perry HD, et al. 2007. Methicillin-resistant Staphylococcus aureus infectious keratitis following refractive surgery. Am J Ophthalmol, 143:629-34.

van Endt JJ, Veraart HG, Kramer R, et al. 1997. A comparison of two ophthalmic steroid-antibiotic combinations after cataract surgery. Eur J Ophthalmol, 7:144-8.

Yee RW, Setabutr P, Foltermann MO, et al. 2006. The effects of topical moxifloxacin $0.5 \%$ ophthalmic solution and gatifloxacin $0.3 \%$ solution on corneal healing after bilateral photorefractive keratectomy. Cornea, 25(Suppl 2):S8-S11.

Yulek F, Ozdek S, Gurelik G, et al. 2006. Effect of topical steroids on corneal epithelial healing after vitreoretinal surgery. Acta Ophthalmol Scand, 84:319-22. 